\title{
WHEN ARE THE ZERO-ENERGY SOLUTIONS TO THE SCHRÖDINGER EQUATION BOUNDED AT INFINITY?
}

\section{A. G. RAMM}

Department of Mathematics

Kansas State University

Manhattan

KS 66506-2602

USA

e-mail: ramm@math.ksu.edu

\begin{abstract}
Conditions for the boundedness of the solutions to equation $-u^{\prime \prime}+q(x) u=0$, $x \in \mathbb{R}$, are given. The function $q(x)$ is assumed compactly supported realvalued bounded and locally integrable.
\end{abstract}

\section{Introduction}

Consider the one-dimensional equation

$$
-u^{\prime \prime}+q(x) u=0, \quad x \in \mathbb{R}, \quad \mathbb{R}:=\{x:-\infty<x<\infty\} .
$$

Assume that

$$
\max _{x \in \mathbb{R}}|q(x)| \leq Q, \quad q(x)=0 \text { for }|x| \geq R .
$$


The questions discussed in this paper are:

(a) When are $u( \pm \infty)$ bounded?

(b) When do the limits $u( \pm \infty)$ exist?

These questions are of practical interest, but, it seems, were not considered earlier. A closely related question is:

Under what condition on a potential the Schrödinger operator in $\mathbb{R}^{3}$ does not have zero as an eigenvalue?

This question was studied and answered in papers [1], [2].

\section{Approach}

Equation (1.1) is equivalent to the integral equation

$$
u(x)=a+b x+\int_{-R}^{x}(x-s) q(s) u(s) d s:=a+b x+V u,
$$

where $a, b$ are constants. Thus, $u(-\infty)$ exists and is bounded if and only if $b=0$, and $u(\infty)$ exists and is bounded if and only if the following relation holds:

$$
\int_{-R}^{R} q(s) u(s) d s=0
$$

If relation (2.2) holds and $b=0$, then

$$
u(-\infty)=a, \quad u(\infty)=a-\int_{-R}^{R} s q(s) u(s) d s
$$

Given $a$, one can uniquely solve the Volterra integral equation (2.1) with $b=0$ and then check if condition (2.2) holds for $u$. The solution to the Volterra equation (2.1) with $b=0$ is

$$
u=a \sum_{j=0}^{\infty} V^{j} 1
$$


Thus, condition (2.2) can be written as

$$
\int_{-R}^{R} q(s) \sum_{j=0}^{\infty} V^{j} 1 d s=\sum_{j=0}^{\infty} \int_{-R}^{R} q(s) V^{j} 1 d s=0 .
$$

Let us formulate the result.

Theorem 1. Let assumption (1.2) hold. Then the limits $u( \pm \infty)$ of any solutions to Equation (1.1) exist and are bounded if and only if $b=0$ and condition (2.5) holds.

Remark 1. Condition $b=0$ is not difficult to check numerically: if $x<-R$, then $u=a+b x$, so $b=0$ if and only if $u(x)$ is a constant when $x$ changes in the region $x<-R$.

Condition (2.5) is difficult to check numerically: since computers have finite precision it is difficult (theoretically impossible) to check numerically if an integral is equal to zero exactly. Practically, it means that an arbitrarily small variation of $q$ may lead to solutions unbounded at infinity.

\section{Problem on Half-Axis}

In scattering theory on half-axis $\mathbb{R}_{+}=[0, \infty)$, one has Equation (1.1) on $\mathbb{R}_{+}$, the solution satisfies the boundary condition

$$
u=0,
$$

and the questions are:

(c) When does $u(\infty)$ exist?

(d) When is $u(\infty)<\infty$ ?

To answer these questions, let us write an integral equation

$$
u(x)=b x+\int_{0}^{x}(x-s) q u d s, \quad b=\text { const }
$$


equivalent to differential equation (1.1) on $\mathbb{R}_{+}$with the boundary condition (3.1). A necessary and sufficient condition for $u(\infty)$ to exist and be finite is

$$
b+\int_{0}^{R} q u d s=0
$$

If condition (3.3) holds, then

$$
u(\infty)=-\int_{0}^{R} s q u d s
$$

One can write the unique solution to (3.2) as

$$
u=b \sum_{j=0}^{\infty} V^{j} x, \quad V f:=\int_{0}^{x}(x-s) q(s) f(s) d s
$$

and condition (3.3) as

$$
1+\sum_{j=0}^{\infty} \int_{0}^{R} q(s)\left(V^{j} s\right) d s=0 .
$$

This is a necessary and sufficient condition for $u(\infty)$ to exist and be finite. To check if condition (3.3) holds exactly is numerically impossible. Therefore, this condition shows that an arbitrarily small perturbation of $q$ may lead to a solution $u$ such that $|u(\infty)|=\infty$.

The two references deal with the conditions under which zero is not an eigenvalue of a Schrödinger operator.

\section{References}

[1] A. G. Ramm, Sufficient conditions for zero not to be an eigenvalue of the Schrödinger operator, Journal of Mathematical Physics 28(6) (1987), 1341-1343.

[2] A. G. Ramm, Conditions for zero not to be an eigenvalue of the Schrödinger operator, II, Journal of Mathematical Physics 29(6) (1988), 1431-1432. 